\title{
HDAC Inhibitors Restore the Capacity of Aged Mice to Respond to Haloperidol through Modulation of Histone Acetylation
}

\author{
Janitza L Montalvo-Ortiz', Jack Keegan', Christopher Gallardo', Nicolas Gerst', Kazuhiro Tetsuka ${ }^{2}$, \\ Chris Tucker', Mitsuyuki Matsumoto ${ }^{2}$, Deyu Fang ${ }^{3}$, John G Csernansky' and Hongxin Dong*, \\ 'Department of Psychiatry and Behavioral Sciences, Northwestern University Feinberg School of Medicine, Chicago, IL, USA; ${ }^{2}$ Astellas Research \\ Institute of America, Skokie, IL, USA; ${ }^{3}$ Department of Pathology, Northwestern University Feinberg School of Medicine, Chicago, IL, USA
}

\begin{abstract}
Antipsychotic drugs are widely prescribed to elderly patients for the treatment of a variety of psychopathological conditions, including psychosis and the behavioral disturbances associated with dementia. However, clinical experience suggests that these drugs may be less efficacious in the elderly individuals than in the young. Recent studies suggest that aging may be associated with epigenetic changes and that valproic acid (VPA), a histone deacetylase inhibitor, may reverse such changes. However, it is not yet known whether HDAC inhibitors can modulate age-related epigenetic changes that may impact antipsychotic drug action. In this study, we analyzed conditioned avoidance response (CAR) and c-Fos expression patterns to elucidate the effect of HDAC inhibitors VPA and entinostat (MS-275) on behavioral and molecular markers of the effects of haloperidol $(H A L)$ in aged mice. Our results showed that HAL administration failed to suppress the avoidance response during the CAR test, suggesting an age-related decrease in drug efficacy. In addition, HAL-induced c-Fos expression in the nucleus accumbens shell and prefrontal cortex was significantly lower in aged mice as compared with young mice. Pretreatment with VPA and MS-275 significantly improved HAL effects on the CAR test in aged mice. Also, VPA and MS-275 pretreatment restored HAL-induced increases in c-Fos expression in the nucleus accumbens shell and prefrontal cortex of aged mice to levels comparable with those observed in young mice. Lastly, but most importantly, increases in c-Fos expression and HAL efficacy in the CAR test of the HAL + VPA and HAL + MS-275 groups were correlated with elevated histone acetylation at the c-fos promoter region in aged mice. These findings suggest that pretreatment with VPA or MS-275 increases the behavioral and molecular effects of HAL in aged mice and that these effects occur via modulation of age-related histone hypoacetylation in the nucleus accumbens shell and prefrontal cortex.

Neuropsychopharmacology (2014) 39, |469-I478; do::I0.1038/npp.2013.346; published online 22 January 20 I4
\end{abstract}

Keywords: aging; haloperidol; epigenetics; valproic acid (VPA); entinostat (MS-275); mice

\section{INTRODUCTION}

Elderly individuals ( $\geqslant 65$ years) represent approximately $12 \%$ of the population in the United States, and one of every five Americans (20\%) will be a senior citizen within the next 20 years. Antipsychotic drugs are often among the medications prescribed to elderly patients to control symptoms of psychosis and the behavioral disturbances associated with dementia. Unfortunately, these medications appear to be less effective and produce more serious side effects in such older individuals (Lonergan et al, 2002).

*Correspondence: $\mathrm{Dr} \mathrm{H}$ Dong, Department of Psychiatry and Behavioral Sciences, Northwestern University Feinberg School of Medicine, 303 E. Chicago Avenue, Ward 12-369, Chicago, IL 606II, USA, Tel: +312503 3433, Fax: +3125030466,

E-mail: h-dong@northwestern.edu

Received 13 June 2013; revised 5 December 2013; accepted 8 December 2013; accepted article preview online 24 December 2013
The therapeutic properties of antipsychotic drugs are mediated by gene expression and signal transduction mechanisms. Both typical and atypical antipsychotics induce the expression of immediate-early genes, such as $c$-fos, in a brain region-specific manner (de Bartolomeis et al, 2013; Kontkanen et al, 2002; Murphy and Feldon, 2001). Regional patterns of c-Fos expression also reflect patterns of neuronal activity associated with the drug response. Specifically, induction of c-Fos expression in the nucleus accumbens and prefrontal cortex has long been used as a marker of neuronal activity and downstream genomic changes associated with antipsychotic drug action (Semba et al, 1996; Sumner et al, 2004).

Aging and age-related diseases have been associated with site-specific changes in the genome that are mediated by epigenetic mechanisms (Ben-Avraham et al, 2012; Houston et al, 2013; Ricobaraza et al, 2009). For example, studies have found that patterns of DNA methylation and histone modification change with age (Das and Tyler, 2012; 
Numata et al, 2012). Alterations of histone acetylation in the hippocampus has been related to memory disturbances that occur with aging in animals (Peleg et al, 2010). In addition, histone deacetylase (HDAC) inhibitors have been used as adjunctive treatments for several neuropsychiatric disorders (Graff et al, 2012; Kwok, 2010). Valproic acid (VPA), a class I HDAC inhibitor, is primarily used not only as a mood stabilizer but also to augment the efficacy of antipsychotic drugs in patients with schizophrenia (Basan et al, 2004). More specific HDAC inhibitors, such as MS-275, are currently being developed as treatments for different types of cancers (Juergens et al, 2011; Witta et al, 2012). However, preclinical studies have suggested that MS-275 may also be useful for increasing the behavioral effects of antipsychotic drugs (Simonini et al, 2006). In this study, we determined whether HDAC inhibitors VPA and MS-257 can restore the efficacy-like effects of HAL in aged mice by modulating histone acetylation at the $c$-fos promoter in the nucleus accumbens shell and prefrontal cortex.

\section{MATERIALS AND METHODS}

\section{Animals}

Young (2-3-months old) and aged (22-24-months old) C57BL/6 male mice ( $n=6-8 /$ group) were obtained from Charles River Laboratories and the National Institute of Aging stock located in Charles River Laboratories (Bar Harbor, Maine). Animals were maintained in a temperature-controlled facility at $60 \%$ relative humidity and $20-21{ }^{\circ} \mathrm{C}$ on a 12 -h light/dark cycle. Food and water were available ad libitum. All procedures were performed in accordance with the NIH guidelines for the treatment of rodents and the Current Guide for the Care and Use of Laboratory Animals under a protocol approved by the Northwestern University Animal Studies Committee. Animal care was taken to minimize the pain, suffering, and the number of animals used.

\section{Drugs}

All drugs used were purchased from Sigma (St Louis, MO), and every dose was freshly prepared before administration. Haloperidol (HAL, $0.01 \mathrm{mg} / \mathrm{kg}$ ) was first dissolved in a small amount $(50 \mu \mathrm{l})$ of glacial acetic acid and made to the final dose volume in $0.9 \%$ saline and the $\mathrm{pH}$ was adjusted to 6.0 with $0.1 \mathrm{M} \mathrm{NaOH}$. VPA (415 mg/kg) was dissolved in $0.9 \%$ saline with $\mathrm{pH}$ 7.3. Entinostat (MS-275, $10 \mathrm{mg} / \mathrm{kg}$ ) was dissolved in DMSO. All compounds and vehicles (saline $0.9 \%$ ) were administered via the intraperitoneal (i.p.) route in a constant volume of $100 \mu \mathrm{l} / 10 \mathrm{~g}$ of body weight. Drugs were administered once a day for 14 consecutive days. VPA and MS-275 were injected 30 min before HAL administration. Behavioral, pharmacokinetic, and cellular/molecular studies were conducted $30 \mathrm{~min}$ after the last injection.

\section{Behavioral Tests}

Conditioned avoidance response (CAR). The CAR procedure test was performed based on previously published protocols (Darvas et al, 2011; Dong et al, 2009; Watt et al, 2013), with slight modifications. Thirty minutes after the last drug administration, mice were placed in a two-way active avoidance (shuttlebox) apparatus (No. EI0-16SC, Coulbourn Instruments) with two equal chambers separated by an open doorway. Upon presentation of a $80-\mathrm{dB}$ white noise conditioned stimulus (CS), the mice had $10 \mathrm{~s}$ to move from one compartment of the shuttlebox into the other. If the mice remained in the same compartment for $>10 \mathrm{~s}$, the unconditioned stimulus (UCS), an intermittent electric foot shock $(0.5 \mathrm{~mA}$ of $0.5 \mathrm{~s} ; 2 \mathrm{~s}$ of intershock interval), was presented in the grid floor until an escape was performed. If the animal did not respond during the $30 \mathrm{~s}$ of the shock period, the trial was terminated (escape failure). Inter-trial intervals varied between 20 and $40 \mathrm{~s}$. The animals underwent 20 trials for 4 consecutive days. The following variables were recorded: avoidance (response to CS within $10 \mathrm{~s}$ ), escape (response to CS and UCS within $30 \mathrm{~s}$ ), and escape failure (failure to respond). All data were shown as percentages (\%) of avoidance, which was the ratio of number of avoidance responses to the total number of trials multiplied by 100 .

\section{Plasma and Brain Concentrations of HAL}

The plasma and brain concentration of HAL was measured using LC-MS/MS (Prominence 2000 series liquid chromatography system, Shimadzu, Kyoto, Japan coupled to an API-5000 mass spectrometer, Applied Biosystems, Foster, CA). A HAL standard curve using $30 \mu \mathrm{l}$ of serially diluted HAL in acetonitrile into $30 \mu \mathrm{l}$ of blank plasma or blank $\times 5$ brain homogenates with final concentration range from 0.01 to $30 \mathrm{ng} / \mathrm{ml}$ plasma (or $\mathrm{ng} / \mathrm{g}$ brain homogenate) was used to determine HAL concentration in the plasma or brain in mice dosed at $0.01 \mathrm{mg} / \mathrm{kg}$. Following protein precipitation by acetonitrile containing $0.1 \%$ of formic acid and $0.1 \mu \mathrm{g} / \mathrm{ml}$ of diazepam (internal standard), samples were cleared by centrifugation ( $\left.15 \mathrm{~min}, 3500 \mathrm{rpm}, 4^{\circ} \mathrm{C}\right)$ and fractionated on a XBridge C18 column $\left(5 \mu \mathrm{m}, 2.1 \times 50 \mathrm{~mm}^{2}\right.$; Waters Corporation, Milford, MA) using a $0.1 \%$ formic acid/water to $0.1 \%$ formic acid/acetonitrile gradient with a flow rate of $0.3 \mathrm{ml} / \mathrm{min}$. Detection of HAL and diazepam was carried out by positive electrospray ionization mode with transitions of $\mathrm{m} / z$ 376.3-123.1 and $\mathrm{m} / \mathrm{z} 285-193$, respectively. The ratio of chromatographic peak areas of HAL to diazepam was used to calculate the HAL concentration. Brain concentration was calculated by multiplying dilution factor of five to brain homogenate concentration.

\section{Immunohistochemistry}

The procedures for c-Fos immunohistochemical staining followed the published protocols (Deutch et al, 1992; Zhao and Li, 2010). Thirty minutes after drug administration, animals were deeply anesthetized and perfused transcardially with $4 \%$ paraformaldehyde. Brains were removed, post-fixed and cut into $40-\mu \mathrm{m}$ thick sections in the coronal plane using a cryostat (Leica CM 1850 UV; Leica, Nussloch, Germany). Approximately 80 sections from each animal were divided into equal quarters in a systemic-uniformrandom manner, and one set of 20 sections was used for c-Fos immunohistochemical staining. Selected brain sections were incubated with a rabbit polyclonal anti-c-Fos antibody (Santa Cruz, 1:20000) overnight at $4{ }^{\circ} \mathrm{C}$. After 
washing, the sections were incubated in biotinylated antirabbit secondary antibody $(1: 200)$ for $2 \mathrm{~h}$ (Vectastain Elite $\mathrm{ABC}$ Kit, Vector Laboratories) and then in an avidin-biotin complex for $1 \mathrm{~h}$ at room temperature. c-Fos-positive cells were visualized using a DAB kit (Vector Laboratories). As a control, the primary antibody was substituted with normal rabbit serum. For the counting of c-Fos-positive cells, photomicrographs were captured with a digital camera using $\times 40$ magnification. The brain regions selected for analysis include the nucleus accumbens shell and the prefrontal cortex, located at Bregma $1.80 \mathrm{~mm}$ based on the mouse atlas (Paxinos and Franklin, 2008). Positive c-Fos cells were characterized by clear dark-brown-labeled, round or oval nuclei. Cell counts were made within a $680 \times 510 \mu \mathrm{m}^{2}$ unit area using the Image J software (US National Institutes of Health). c-Fos-positive cells were averaged to obtain the final mean \pm SEM.

\section{Western Blotting}

Nucleus accumbens shell and prefrontal cortex tissue samples were collected from $300 \mu \mathrm{m}$ coronal mouse brain cryosections using a 1-mm microdissection punch. Collected tissue was then frozen and stored at $-80{ }^{\circ} \mathrm{C}$ until further use. Brain tissue was homogenized in 3\% (w/v) icecold T-PER tissue protein extraction reagent (Thermo Scientific). The homogenates were centrifuged at $20000 \mathrm{~g}$ for $10 \mathrm{~min}$ at $4{ }^{\circ} \mathrm{C}$, and the supernatants were used for immunoblotting. Protein content was measured using the BCA protein assay kit (Thermo Scientific) according to the manufacturer's instructions. Samples were separated on $8-15 \%$ Bis-Tris gel and transferred onto a nitrocellulose membrane (Invitrogen). Blots were blocked and immunostained overnight at $4{ }^{\circ} \mathrm{C}$ with primary antibody against c-Fos (Santa Cruz) or $\beta$-actin (Santa Cruz) in blocking buffer. The membrane was then incubated with a $1: 3000$ dilution of HRP-conjugated secondary goat anti-mouse or anti-rabbit antibodies for $2 \mathrm{~h}$ at room temperature. Blotted protein was detected and quantified using the ChemiDocMC Imaging System (Bio-Rad) and Image J Software. Levels of target protein expression were normalized to $\beta$-actin.

\section{Chromatin Immunoprecipitation Assay}

The commercially available Magna ChIP G Tissue Kit (17-20000, Millipore) was used. Fragmented chromatin lysate (400-600 bp) was immunoprecipitated with $5 \mu \mathrm{g}$ of antibody directed against histone H3 acetyl Lys 27 (H3K27ac, Active Motif) and histone H4 acetyl Lys (H4K12ac, Millipore). After immunoprecipitation, the DNA-histone complex was incubated with salmon sperm DNA/protein A-agarose beads for $1 \mathrm{~h}$ for non-specific binding. The DNA-histone complex was eluted from the beads with $\mathrm{NaHCO} 3 / \mathrm{SDS}$ and dissociated at $65{ }^{\circ} \mathrm{C}$ for $4 \mathrm{~h}$ under high-salt conditions. Proteins were digested using proteinase $\mathrm{K}$ treatment. The DNA, associated with acetylated histones, was precipitated with $100 \%$ ethanol and resuspended in $80 \mu \mathrm{l}$ of PCR-grade water.

\section{Quantitative Real-Time PCR}

Levels of specific histone modifications at the promoter regions of genes were measured using primers targeting c-fos (forward: 5'-GCGATTGCAGCTAGCAACTGAGAA-3', reverse: 5'-CGCGTTGAAACCCGAGAACATCAT-3' amplified region $140 \mathrm{bp}$ upstream of the start codon) and $\beta$-actin (forward: 5'-GCGTCCACCCGCGAGTACAA-3', reverse: $5^{\prime}$-TCCATGGCGAACTGGTGGCG-3') as our control inputs, and immunoprecipitated DNA amplification reactions were run in triplicates in the presence of SYBR Green (Applied Biosystems). Fold differences were determined by raising 2 to the power of $\Delta \mathrm{Ct}$.

\section{Statistical Analysis}

All data are expressed as mean \pm SEM. Two-way analysis of variance (ANOVA) was used to assess the effects of age and drug administration (treatment) on avoidance response, HAL concentrations in the plasma and brain, levels of acetylation of $\mathrm{H} 3 \mathrm{~K} 27$ and $\mathrm{H} 4 \mathrm{~K} 12$ at $c$-fos promoter and c-Fos-positive cells and protein levels in the nucleus accumbens shell and prefrontal cortex. Post-hoc differences were assessed using Bonferroni's post-hoc test only when a significant main effect or interaction was found. The level of statistical significance was set as $p<0.05$.

\section{RESULTS}

\section{Pretreatment with HDAC Inhibitors Improves HAL Efficacy in Aged Mice}

There was a significant effect of treatment $\left(\mathrm{F}_{5,70}=13.32\right.$, $p<0.0001)$ and an age $\times$ treatment interaction $\left(\mathrm{F}_{5,70}=\right.$ 2.996, $p<0.01)$ but no effect of age $\left(\mathrm{F}_{1,70}=1.439, p=\right.$ $0.2344)$ in the percentage (\%) of avoidance response in the CAR test. Bonferroni's post-hoc analysis revealed a significant decrease in the percentage of avoidance response during Trial $2(p<0.05)$, Trial $3(p<0.05)$, and Trial 4 $(p<0.05)$ of HAL-treated young mice compared with salinetreated young mice (Figure $1 \mathrm{a}$ and b). Similarly, a significant decrease was observed in the percentage of avoidance response during Trial $2(p<0.05)$, Trial 3 $(p<0.05)$ and Trial $4(p<0.05)$ of HAL + VPA- and HAL + MS-275-treated young mice compared with saline-treated young mice (Figure $1 \mathrm{a}$ and $\mathrm{b}$ ). In aged mice, no differences were found in HAL-treated aged mice relative to salinetreated aged mice across the different trials in the CAR test, suggesting that the efficacy of HAL is decreased in aged mice. However, a significant decrease in the percentage of avoidance was observed during Trial $2(p<0.001)$, Trial 3 $(p<0.01)$, and Trial $4(p<0.01)$ of HAL + VPA-treated mice (Figure 1c) and Trial $2(p<0.01)$, Trial $3(p<0.001)$, and Trial $4(p<0.001)$ of HAL + MS-275-treated (Figure 1d) aged mice relative to saline- or HAL-treated aged mice. These results indicate that pretreatment of VPA and MS-275 decrease the avoidance response in HAL-treated aged mice, suggesting that HDAC inhibitors may restore the efficacy of HAL in aging.

\section{Lack of Age-Related Changes of HAL Levels in the Plasma and Brain}

To exclude the possibility that any observed effects of age on the efficacy of HAL is due to pharmacokinetic changes in the body or brain, HAL concentrations in plasma and brain 

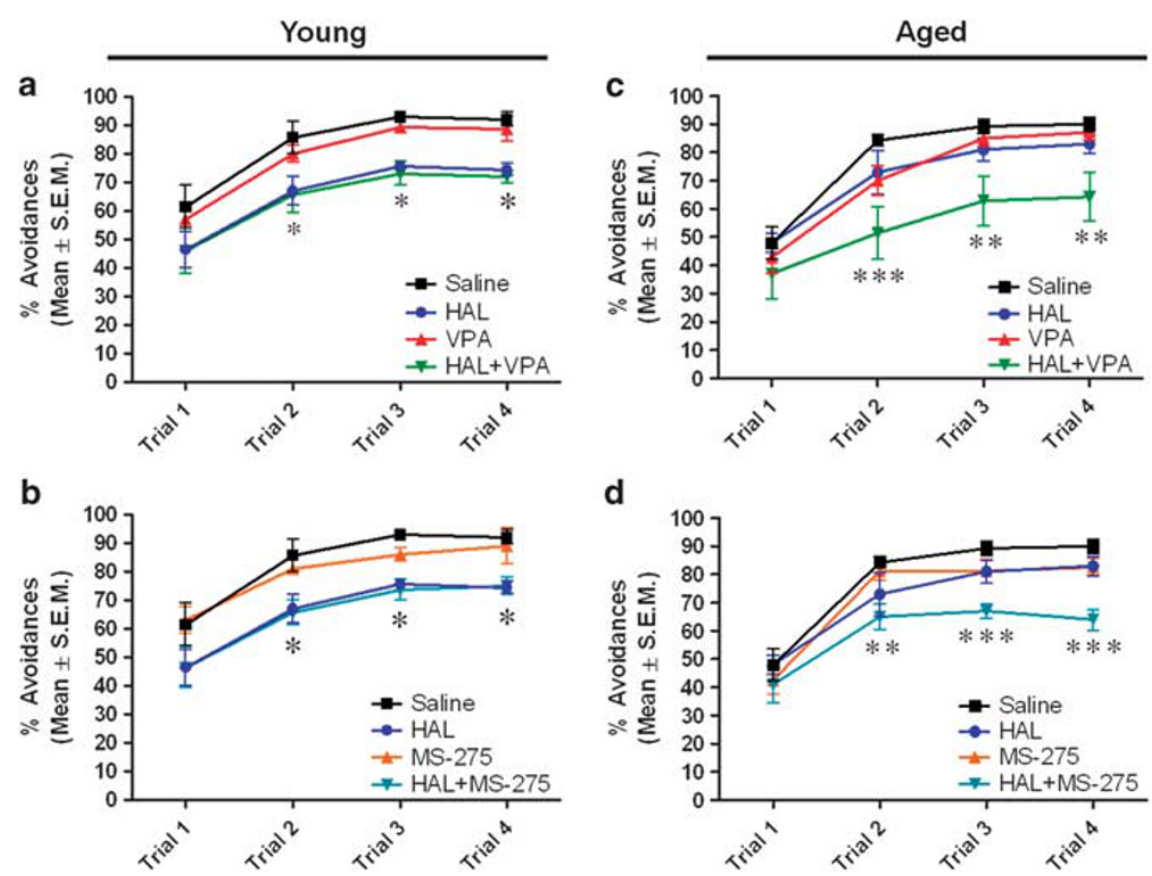

Figure I Effect of pre-treatment of HDAC inhibitors on conditioned avoidance response in HAL-treated young and aged mice. Data are shown as the percentage of avoidance response of young ( $\mathrm{a}$ and $\mathrm{b}$ ) and aged mice ( $\mathrm{c}$ and $\mathrm{d}$ ). Data are presented as mean \pm SEM ( $n=6-8$ per group). In panel a, * $p<0.05$ vs the saline-, VPA-treated groups. In panel b, * $p<0.05$ vs the saline-, MS-275-treated groups. In panel c, *** $p<0.0$ I, **** $p<0.00$ I vs the saline-, HAL-, and VPA-treated groups. In panel d, ** $p<0.01$, *** $p<0.00$ I vs the saline-, HAL-, and MS-275-treated groups.

Table I Measurement of HAL Concentration in Brain and Plasma Samples of Young and Aged Mice

\begin{tabular}{llccc}
\hline Age, months & Drug treatment & Brain concentration, ng/g & Plasma concentration, ng/ml & Brain:plasma ratio \\
\hline $2-3$ & HAL & $21.05 \pm 1.34$ & $6.38 \pm 0.48$ \\
& HAL + VPA & $27.25 \pm 4.34$ & $6.09 \pm 1.04$ & 3.30 \\
& HAL + MS-275 & $20.63 \pm 7.78$ & $6.30 \pm 2.21$ & 4.47 \\
$22-24$ & HAL & $28.08 \pm 4.53$ & $6.48 \pm 1.11$ & 3.27 \\
& HAL + VPA & $27.03 \pm 1.73$ & $5.56 \pm 1.07$ & 4.33 \\
& HAL + MS-275 & $24.18 \pm 4.88$ & $6.86 \pm 1.29$ & 3.86 \\
\end{tabular}

Brain and plasma concentrations were assessed on the last day of treatment, 30 min after the last dose. Brain:plasma ratio was calculated as the ratio of brain to plasma HAL concentration. No significant differences were found between the young and aged mice groups in HAL concentration of the brain and plasma and brain:plasma ratio. Data represent mean \pm SEM $(n=4-5)$.

samples from young and aged mice were measured (Table 1). Plasma and brain HAL concentrations were within the expected ranges in both the young and aged mice groups. Two-way ANOVA analysis revealed no significant effect of age $\left(\mathrm{F}_{1,18}=4.19, p=0.1648\right)$, treatment $\left(\mathrm{F}_{1,18}=\right.$ $5.12, p=0.1833)$, or an age $\times$ treatment interaction $\left(\mathrm{F}_{1,18}=\right.$ 2.73, $p=0.3201$ ) on plasma HAL concentrations. Also, there was no significant effect of age $\left(\mathrm{F}_{1,18}=1.16, p=0.7411\right)$, treatment $\left(\mathrm{F}_{1,18}=0.23, p=1.1635\right)$, or an age $\times$ treatment interaction $\left(\mathrm{F}_{1,18}=0.71, p=0.5124\right)$ on brain HAL concentrations. Additionally, brain:plasma ratios were measured to examine changes in CNS permeability of HAL under different conditions. In general, compounds with a brain:plasma ratio $>1$ are considered to have good CNS penetration. In our study, brain:plasma ratios were $>1$ across all age and treatment groups. Two-way ANOVA analysis found no significant effect of age $\left(\mathrm{F}_{1,18}=0.85, p=0.6995\right)$, treatment $\left(\mathrm{F}_{1,18}=0.7841, p=0.9521\right)$, or an age $\times$ treatment interaction $\left(\mathrm{F}_{1,18}=0.25, p=1.1421\right)$ on brain:plasma ratios.

\section{Pretreatment with HDAC Inhibitors Increases c-Fos-Positive Cells and Protein Levels in the Nucleus Accumbens Shell and Prefrontal Cortex of HAL-Treated Aged Mice}

Immunohistochemical staining. We used an immunohistochemical approach to examine how age and treatment affected c-Fos protein expression in the nucleus accumbens shell and the prefrontal cortex (Figure 2).

Nucleus accumbens shell. Two-way ANOVA showed a significant effect of treatment $\left(\mathrm{F}_{5,70}=49.85, p<0.0001\right)$ and an age $\times$ treatment interaction $\left(\mathrm{F}_{5,70}=9.54, p<0.0001\right)$ but no effect of age $\left(F_{1,70}=1.03, p=0.3127\right)$. Bonferroni 


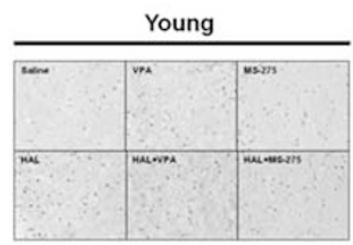

b

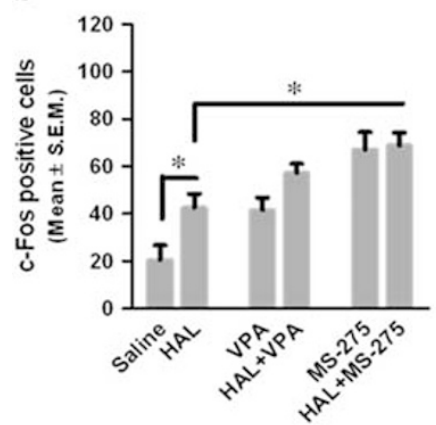

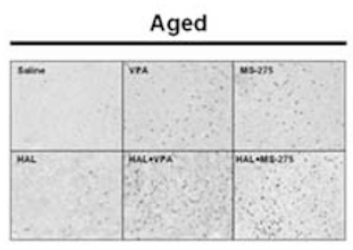

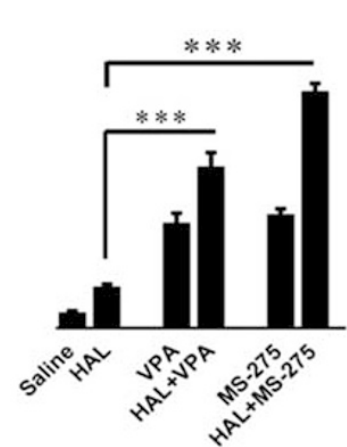

C

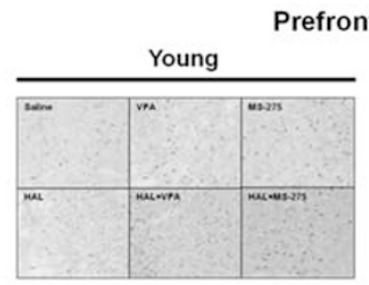

d

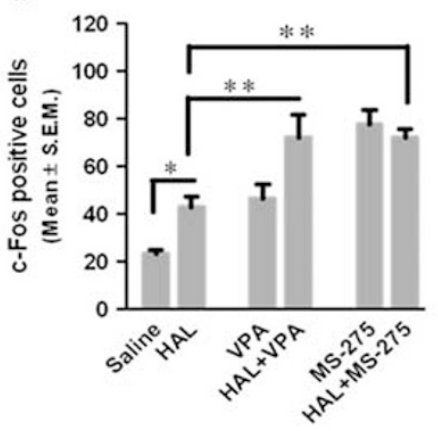

Figure 2 Effect of pretreatment of HDAC inhibitors on c-Fos-positive cells in the nucleus accumbens shell and prefrontal cortex of HAL-treated young and aged mice. Representative photomicrographs of c-Fos-positive cells in the nucleus accumbens shell (a) and prefrontal cortex (c) are shown for the saline, HAL-, VPA-, HAL + VPA-, MS-275-, and HAL + MS-275-treated groups of young and aged mice. Quantitative analysis of c-Fos-positive cells counting is shown for both young (gray bars) and aged (black bars) mice of the nucleus accumbens shell (b) and prefrontal cortex (d). Data are presented as mean \pm SEM ( $n=6-8$ per group). $* p<0.05$, $* * * 0.01$, **** $p<0.001$.

post-hoc tests showed that c-Fos-positive cells were significantly increased in HAL-treated young mice relative to saline-treated aged mice. This increase in c-Fos-positive cells was not observed in HAL-treated aged mice when compared with saline-treated aged mice. In young mice groups, HAL + MS-275-treated $(p<0.05)$, but not $\mathrm{HAL}+$ VPA-treated, mice was associated with significant increases in c-Fos-positive cells relative to HAL-treated group. In aged mice groups, HAL + VPA $(p<0.001)$ and HAL + MS$275(p<0.001)$ significantly increase c-Fos-positive cells as compared with either saline or HAL treatments (Figure $2 \mathrm{a}$ and $\mathrm{b})$.

Prefrontal cortex. Two-way ANOVA analysis showed a significant effect of age $\left(\mathrm{F}_{5,82}=29.25, p<0.0001\right)$, treatment $\left(\mathrm{F}_{5,82}=47.66, p<0.0001\right)$, and an age $\times$ treatment interaction $\left(\mathrm{F}_{5,82}=10.46, p<0.0001\right)$. Bonferroni's post-hoc tests showed a significant increase in $c$-Fos protein levels in young mice treated with HAL $(p<0.05)$ when compared with saline. This increase in c-Fos-positive cells after HAL treatment was not observed in aged mice. In young mice groups, a significant increase was observed in HAL + VPA $(p<0.01)$ and HAL + MS-275 $(p<0.01)$ treated mice as compared with HAL-treated mice. In aged mice groups, a significant increase in c-Fos-positive cells was observed in $\mathrm{HAL}+\mathrm{VPA} \quad(p<0.001)$ and HAL + MS-275 $(p<0.001)$ treated mice relative to either saline- or HAL-treated mice (Figure 2c and $\mathrm{d}$ ).

\section{Western blotting}

Nucleus accumbens shell. Two-way ANOVA revealed a significant effect of age $\left(\mathrm{F}_{1,24}=10.74, p<0.01\right)$, treatment $\left(\mathrm{F}_{5,24}=28.22, p<0.0001\right)$, and an age $\times$ treatment inter- action $\left(\mathrm{F}_{5,24}=8.54, p<0.0001\right)$. Bonferroni post-hoc tests showed a significant increase in c-Fos protein levels in HAL-treated young mice as compared with saline-treated young mice. This increase in c-Fos protein levels was not observed in HAL-treated aged mice. However, a significant increase was found in HAL + VPA $(p<0.01)$ and HAL + MS-275 $(p<0.001)$ treated mice as compared with either saline- or HAL-treated mice (Figure $3 \mathrm{a}$ and $\mathrm{b}$ ).

Prefrontal cortex. Two-way ANOVA revealed a significant effect of age $\left(\mathrm{F}_{1,42}=7.57, p<0.01\right)$, treatment $\left(\mathrm{F}_{5,42}=15.28, p<0.0001\right)$, and an age $\times$ treatment interaction $\left(\mathrm{F}_{5,42}=2.36, p<0.05\right)$. Bonferroni's post-hoc analysis found a significant increase in c-Fos protein levels in HALtreated young mice relative to saline-treated young mice. This increase was not observed in HAL-treated aged mice when compared with saline-treated aged mice. However, a significant increase in c-Fos protein levels was observed in $\mathrm{HAL}+\mathrm{VPA}(p<0.001)$ and HAL + MS-275 $(p<0.05)$ treated aged mice as compared with HAL-treated aged mice (Figure $3 \mathrm{c}$ and $\mathrm{d}$ ).

\section{Pretreatment with HDAC Inhibitors Reverses Hypoacetylation at the $c$-fos Promoter Region in the Nucleus Accumbens Shell and Prefrontal Cortex in HAL-Treated Aged Mice}

Nucleus accumbens shell. Two-way ANOVA analysis found a significant effect in treatment $\left(\mathrm{F}_{5,32}=71.75\right.$, $p<0.0001), \quad$ age $\times$ treatment interaction $\left(\mathrm{F}_{5,32}=13.64\right.$, $p<0.0001)$ but not in age $\left(F_{1,32}=3.16, p=0.0851\right)$. Bonferroni post-hoc analysis found a significant increase in $\mathrm{H} 3 \mathrm{~K} 27$ acetylation at the $c$-fos promoter in HAL-treated $(p<0.01)$ young mice when compared with saline-treated young mice. 
a

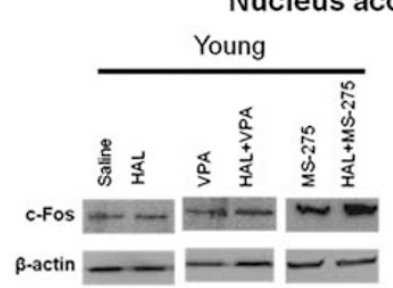

b

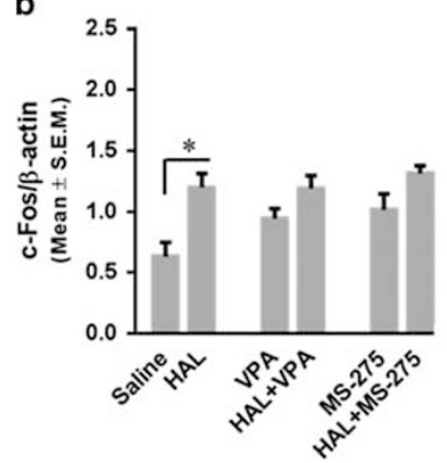

c

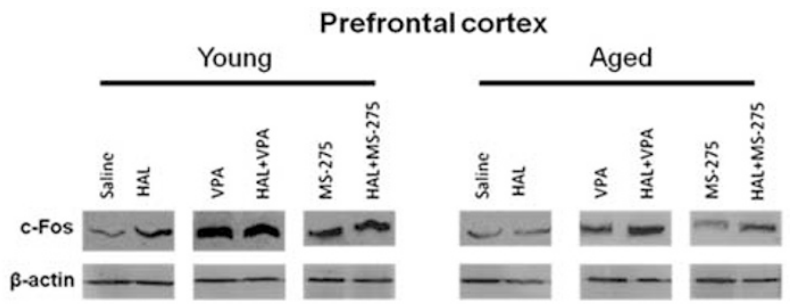

d

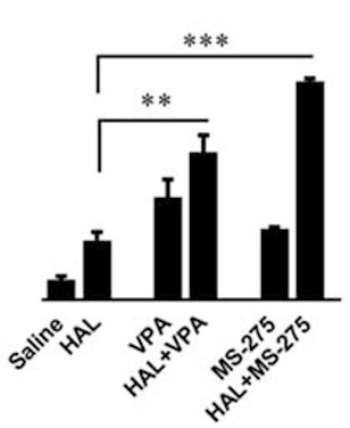

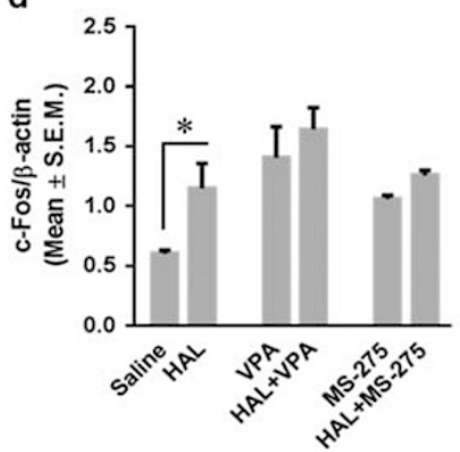

*

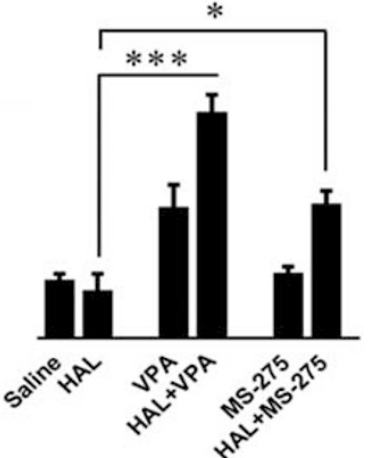

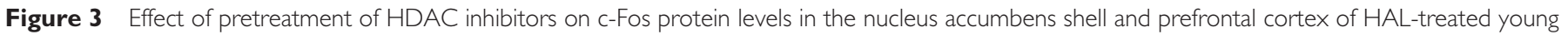

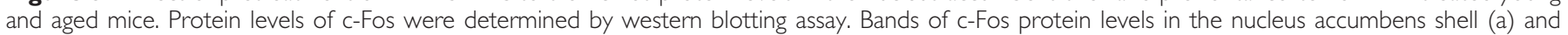

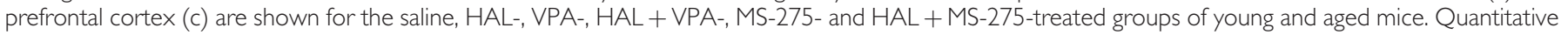

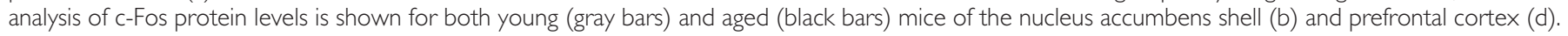
Data are presented as mean \pm SEM ( $n=4-5$ per group). $* p<0.05$, $* * * 0.01$, **** $p<0.001$.

In addition, a significant increase was found in $\mathrm{HAL}+$ MS-275-treated $(p<0.05)$ young mice as compared with HAL-treated young mice. In aged mice groups, no differences in H3K27 acetylation were found between salineand HAL-treated mice. However, a significant increase was observed with HAL + VPA $(p<0.001)$ and HAL + MS-275 $(p<0.001)$ treated aged mice relative to either saline- or HAL-treated aged mice groups (Figure 4a).

In the H4K12, two-way ANOVA analysis found a significant effect in age $\left(\mathrm{F}_{1,24}=274.81, p<0.0001\right)$, treatment $\left(\mathrm{F}_{5,24}=299.46, p<0.0001\right)$, and an age $\times$ treatment interaction $\left(\mathrm{F}_{5,24}=20.57, p<0.0001\right)$. Bonferroni's post-hoc analysis found a significant increase in H4K12 acetylation at the $c$-fos promoter of HAL-treated young mice relative to saline-treated young mice $(p<0.001)$. A significant increase was also observed in HAL + VPA- $(p<0.001)$ and HAL + MS-275- $(p<0.001)$ treated young mice relative to salinetreated young mice. In the aged mice groups, no differences were found between saline and HAL-treated mice. However, a significant increase was observed with HAL + VPA $(p<0.001)$ and HAL + MS-275 $(p<0.001)$ treated aged mice as compared with either saline or HAL-treated aged mice (Figure $4 b$ ).

Prefrontal cortex. Two-way ANOVA revealed a significant effect in treatment $\left(\mathrm{F}_{5,24}=247.84, p<0.0001\right)$ and an age $\times$ treatment interaction $\left(\mathrm{F}_{5,24}=29.31, p<0.0001\right)$ but not in age $\left(F_{1,24}=2.27, p=0.1446\right)$. Bonferroni post-hoc analysis found that a significant increase in $\mathrm{H} 3 \mathrm{~K} 27$ acetylation was observed in HAL-treated aged mice relative to saline-treated young mice $(p<0.001)$. This increase in H3K27 acetylation was not observed in HAL-treated aged mice relative to saline-treated aged mice. However, a significant increase in $\mathrm{H} 3 \mathrm{~K} 27 \mathrm{ac}$ was found in HAL $+\mathrm{VPA}(p<0.001)$ and HAL + MS-275 $(p<0.001)$ treated aged mice when compared with either saline or HAL-treated aged mice (Figure 4c). In the H4K12ac, two-way ANOVA revealed a significant effect in age $\left(\mathrm{F}_{1,24}=339.4, p<0.0001\right)$ but not in treatment $\left(\mathrm{F}_{5,24}=\right.$ 1.851, $p=0.1409)$ and age $\times$ treatment interaction $\left(\mathrm{F}_{5,24}=\right.$ 2.108, $p=2.108$ ). Bonferroni's post-hoc analysis found a significant age-related decrease in saline-, HAL-, HAL + VPA-, VPA-, HAL + MS-275-, and MS-275-treated mice $(p<0.001)$. No differences were found across the different drug treatment groups in either young or aged mice (Figure 4d).

These results show that pretreatment of HDAC inhibitors increase acetylated levels of H3K27 and H4K12 at nucleus accumbens shell, but only at $\mathrm{H} 3 \mathrm{~K} 27 \mathrm{ac}$ of the prefrontal cortex, in HAL-treated aged and young mice. These findings suggest that aging induces hypoacetylation at the $c$-fos promoter of the nucleus accumbens shell and prefrontal cortex, which can be reversed by pretreatment of HDAC inhibitors in HAL-treated aged mice.

To assess whether levels of acetylation at the $c$-fos promoter in the nucleus accumbens shell and prefrontal were related to changes in HAL efficacy (percentage of avoidance response) within individual animals, a Pearson's linear regression analysis was performed. Our results revealed that levels of acetylation in H3K27 at c-fos promoter in the nucleus accumbens shell were directly correlated with the percentage of avoidance response across the different drug treatment groups of aged mice $(r=0.7944, p<0.0001)$ but not of young mice $(r=0.3013$, $p<0.0646$ ) (Figure 5a and $\mathrm{b}$ ). In the prefrontal cortex, levels of acetylation in $\mathrm{H} 3 \mathrm{~K} 27$ were directly correlated with the 


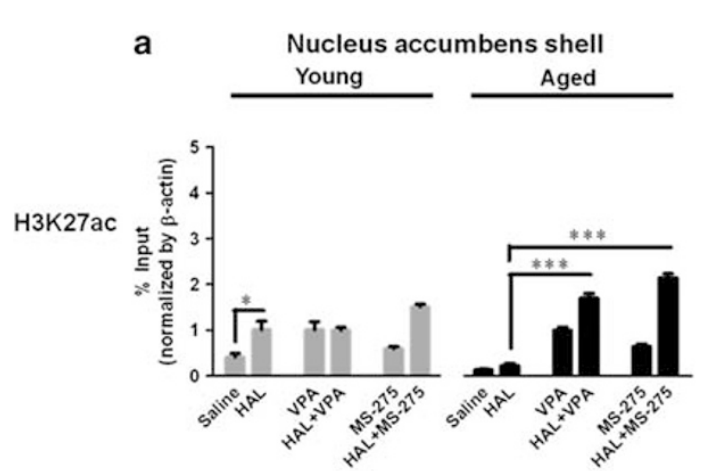

b

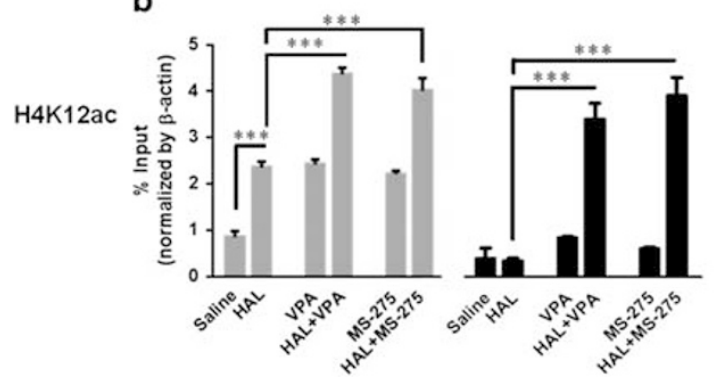

c

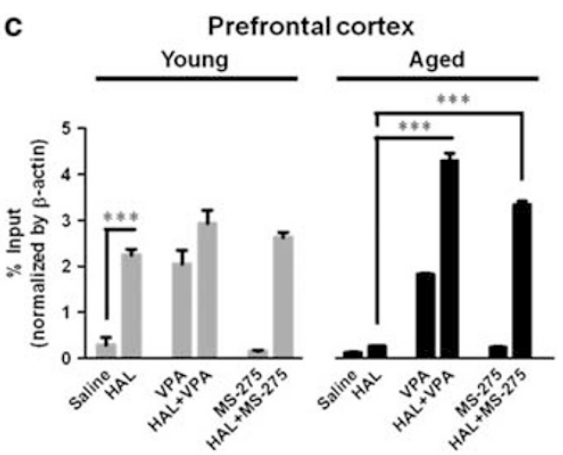

d

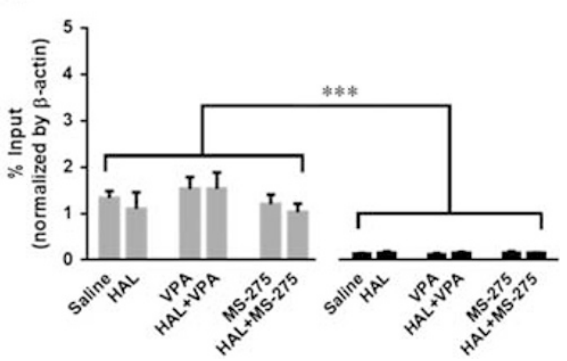

Figure 4 Effect of pretreatment of HDAC inhibitors on the acetylation patterns at the c-fos gene promoter in the nucleus accumbens shell and prefrontal cortex of HAL-treated young and aged mice. Acetylation of H3K27 and H4KI2 at the c-fos promoter of the nucleus accumbens shell (a and b) and prefrontal cortex ( $c$ and d) from young (gray bars) and aged (black bars) mice was measured as the percentage of input with chromatin immunoprecipitation assay. Data are presented as mean \pm SEM, $n=4-5 . * 2<0.05$, *** $p<0.01$, $* * * * 00.001$.
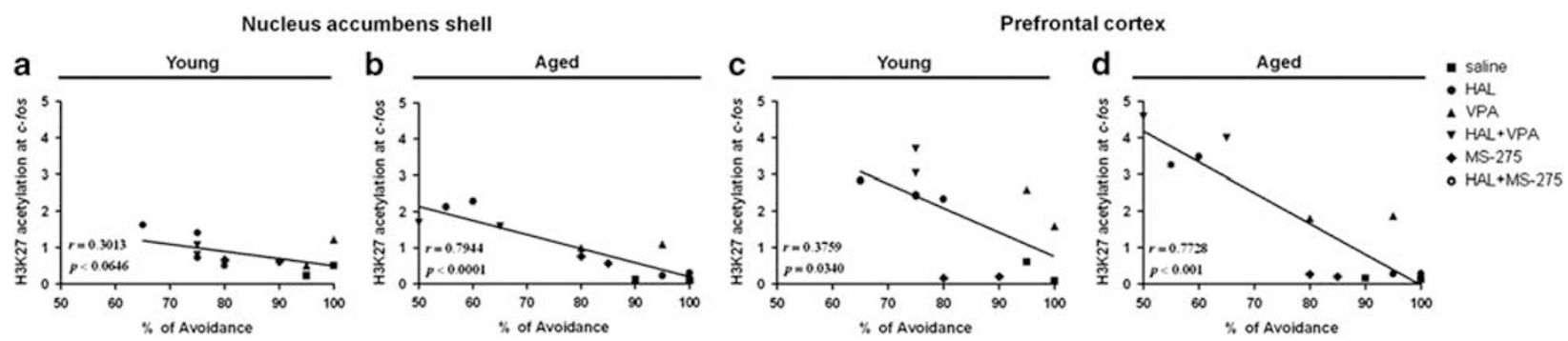

Figure 5 Correlation analysis of acetylated levels of $\mathrm{H} 3 \mathrm{~K} 27$ and $\mathrm{H} 4 \mathrm{~K} / 2$ at the c-fos promoter against the avoidance response of young and aged mice. Correlation analysis was performed by the Pearson's test. Acetylated H3K27 at c-fos promoter of nucleus accumbens shell (a and b) and prefrontal cortex ( $c$ and $d)$ are shown as a function of percentage of avoidance response of young and aged mice. ( $n=3-4$ per group).

percentage of avoidance across the different drug treatment groups of aged mice $(r=0.7728, p=0.001)$ but not of young mice $(r=0.3759, p=0.0340)$ (Figure $5 \mathrm{c}$ and $\mathrm{d}$ ).

Levels of acetylation in $\mathrm{H} 3 \mathrm{~K} 27$ at $c$-fos promoter also correlated with the number of c-Fos-positive cells in the nucleus accumbens shell of aged mice $(r=0.9397$, $p<0.0001)$ but not of young mice $(r=0.1436, p=0.1477)$. H4K12 acetylation was directly correlated with c-Fospositive cells in the nucleus accumbens shell of both young $(r=0.5946, p<0.01)$ and aged $(r=0.5249, p<0.01)$ mice. In addition, c-Fos-positive cells were directly correlated with the percentage of avoidance response in young $(r=0.3544, p<0.01)$ and aged $(0.7311, p<0.0001)$ mice. In the prefrontal cortex, H3K27 acetylation was directly correlated with c-Fos-positive cells in young $(r=0.8989$, $p<0.001)$ and aged $(r=0.8389, p<0.0001)$ mice. H4K12 acetylation was not correlated with c-Fos-positive cells in both young $(r=0.3264, p=0.1391)$ and aged $(r=0.0000$, $p=0.9989)$ mice. In addition, c-Fos-positive cells in the prefrontal cortex were directly correlated with the percentage of avoidance response in young $(r=0.4496, p<0.001)$ and aged $(r=0.05667, p<0.0001)$ mice.

\section{DISCUSSION}

In this study, we investigated how age-related epigenetic alterations impacted the behavioral and molecular effects of the antipsychotic drug HAL and whether HDAC inhibitors could mitigate these effects. Our results suggest that reversal of histone hypoacetylation at the $c$-fos promoter in the nucleus accumbens shell and prefrontal cortex may be a mechanism by which HDAC inhibitors VPA and MS-275 can restore the capacity of aged mice to respond to HAL. 
We evaluated the effects of HDAC inhibitors on HAL efficacy in mice using CAR. CAR paradigm is a wellestablished test for assessing the potential for antipsychotic drug efficacy in preclinical studies (Wadenberg, 2010). Both typical and atypical antipsychotics selectively suppress the avoidance response. HAL, in particular, has been extensively studied using this preclinical model (Guidotti et al, 2009; Kapur, 2003). In our study, we found that the capacity of HAL to suppress the avoidance response was diminished in aged mice.

Antipsychotic drugs also induce the expression of immediate early genes, including $c$-fos, in a brain-regionspecific manner. One of the key neural sites of antipsychotic action is the nucleus accumbens shell (Deutch et al, 1992; Robertson et al, 1994; Semba et al, 1999) as both typical and atypical antipsychotic drug induce c-Fos expression in this brain region. These effects have been related to the therapeutic properties of antipsychotic drugs (Lieberman et al, 2008; Robertson et al, 1994). More recently, the prefrontal cortex has been identified as another brain subregion area that may be involved in antipsychotic efficacy (Wadenberg et al, 2007). C-Fos induction by pharmacological stimuli is reduced in both nucleus accumbens and prefrontal cortex of aged mice (Burke and Barnes, 2006; Nagahara and Handa, 1997). The results of our study replicate and extend these findings.

Epigenetic drift likely occurs during aging and results in altered gene expression patterns that compromise function and cellular adaptations to environmental stimuli (Desjardins et al, 1997), including stimuli that are related to learning and memory (Burke and Barnes, 2006; Tanic et al, 2007). For example, a recent study found that age-related decreases of Arc gene transcription in the hippocampus is mediated by DNA methylation and results in decreased gene transcription, decreased plasticity, and disruptions in memory storage and retrieval processes (Penner et al, 2011). Histone modifications have also been found to have a role in age-related memory impairment. Recent data suggest that decreases in acetylated $\mathrm{H} 4$ lysine 12 (H4K12ac) of learning-induced genes in the aged hippocampus may be associated with memory consolidation deficits (Peleg et al, 2010). These age-related histone acetylation changes and behavioral changes were reversed by the administration of HDAC inhibitors, presumably by increases in the expression of learning-induced genes in the hippocampus. This suggests that histone acetylation may be a critical target for reversing age-related memory deficits. However, whether aging-induced epigenetic modification has a role in responses to psychotropic medications in the brain is not known.

In this study, we found age-related decreases in c-Fos expression in the nucleus accumbens shell and prefrontal cortex of HAL-treated mice that were correlated with histone hypoacetylation at the $c$-fos promoter region. H3K27 and H4K12 acetylation markers were differentially regulated in the nucleus accumbens shell and prefrontal cortex. These brain-region-specific changes in the acetylation of $\mathrm{H} 4 \mathrm{~K} 12$ could reflect age-related decreases in histone acetylation. However, the differences observed in acetylation between the nucleus accumbens shell and prefrontal cortex may be related to the more critical involvement of the nucleus accumbens shell in the mediation of anti- psychotic efficacy. This notion is supported by a study showing that local application of antipsychotics in this brain region significantly suppresses the avoidance response (Deutch et al, 1992; Semba et al, 1999). In other words, elevating the levels of acetylation at the c-fos promoter in the nucleus accumbens shell may be specifically required for an optimal response to antipsychotic drugs.

HDAC inhibitors have been used to increase acetylated levels of genes, thus restoring the expression of genes that are compromised during aging (Peleg et al, 2010; Tang et al, 2011). In addition, preclinical and clinical studies have found that VPA, a class I HDAC inhibitor, can augment the therapeutic effects of antipsychotics (Guidotti et al, 2009; Suzuki et al, 2009). To evaluate whether VPA can also restore the behavioral effects of HAL in aged mice, we used the CAR test and found that pretreatment with VPA restored the ability of HAL to suppress the avoidance response in aged mice and in a manner that was probably associated with the modulation of histone acetylation.

It is noteworthy that VPA can influence neuronal activity through mechanisms other than epigenetic regulation. For example, VPA affects GABAergic and glutamatergic systems, kinase pathways, and gene expression and transcription factors. VPA has also emerged as an anti-neoplastic agent that can influence cell growth, differentiation, and apoptosis. In addition, VPA displays a neuroprotective function in several animal models of neurodegenerative diseases (Nuutinen et al, 2010). However, recent findings suggest that all of these diverse actions of VPA may be mediated through its action as a HDAC inhibitor (Chuang et al, 2009; Monti et al, 2009). In our study, increases in c-Fos expression after pretreatment with VPA were correlated with increased acetylation at the $c$-fos promoter. These results suggest that pretreatment with VPA may have restored c-Fos expression in the nucleus accumbens shell and prefrontal cortex by increasing acetylation levels at the promoter region.

To further support the notion that the restoration of the effects of HAL on c-Fos protein levels in the nucleus accumbens shell and prefrontal cortex is mediated by histone acetylation modulation, we examined an additional HDAC inhibitor MS-275. We again found that pretreatment of MS-275 restored the ability of HAL to suppress the avoidance response in aged mice. Although both compounds are class I HDAC inhibitors, the structure and function of VPA and MS-275 differ. While VPA is a broad-acting class I HDAC inhibitor, MS275 is more selective for HDAC1 $\left(\mathrm{IC}_{50}=300 \mathrm{nM}\right)$ over HDAC3 $\left(\mathrm{IC}_{50}=8 \mu \mathrm{M}\right)$ (Kazantsev and Thompson, 2008; Khan et al, 2008). Additionally, MS-275 may have more potent and long-lasting effects than VPA (Simonini et al, 2006). The future study of even more selective subtypes of class I HDAC inhibitors will allow us to identify specific epigenetic changes that are involved in influencing the efficacy of HAL and other antipsychotic drugs under a variety of physiological conditions. Moreover, future studies involving the study of age-related changes in the brain's dopaminergic system may help to identify the specific molecular mechanisms that underlie how aging may be related to other effects of antipsychotic drugs, such as their acute and chronic neurological side effects.

In conclusion, the results of this study suggest that pretreatment with the HDAC inhibitors VPA and MS-275 
may be a useful strategy for restoring the normative effects of HAL in animals and possibly for augmenting the clinical efficacy in aged patients. Our results also provide new insights into the epigenetic mechanisms that may be involved in mediating the effects of aging on the brain's dopaminergic system.

\section{FUNDING AND DISCLOSURE}

The authors declare no conflict of interest.

\section{ACKNOWLEDGEMENTS}

This work has been funded by National Institutes of Mental Health (R21 MH100919-01A1) to HD, National Center for Research Resources (NCRR) and the National Center for Advancing Translational Sciences (NCATS), National Institutes of Health (NIH) to JLM-O through Grant Number TL1 5TL1RR025739. JGC has served as a Data Safety and Monitoring Board (DSMB) member for Eli Lilly and SanofiAventis and received funding for his research from Genentech. NG, KT, MM, and CT are employees of Astellas Pharma.

\section{REFERENCES}

Basan A, Kissling W, Leucht S (2004). Valproate as an adjunct to antipsychotics for schizophrenia: a systematic review of randomized trials. Schizophr Res 70: 33-37.

Ben-Avraham D, Muzumdar RH, Atzmon G (2012). Epigenetic genome-wide association methylation in aging and longevity. Epigenomics 4: 503-509.

Burke SN, Barnes CA (2006). Neural plasticity in the ageing brain. Nat Rev Neurosci 7: 30-40.

Chuang DM, Leng Y, Marinova Z, Kim HJ, Chiu CT (2009). Multiple roles of HDAC inhibition in neurodegenerative conditions. Trends Neurosci 32: 591-601.

Darvas M, Fadok JP, Palmiter RD (2011). Requirement of dopamine signaling in the amygdala and striatum for learning and maintenance of a conditioned avoidance response. Learn Mem 18: 136-143.

Das C, Tyler JK (2012). Histone exchange and histone modifications during transcription and aging. Biochim Biophys Acta 1819: 332-342.

de Bartolomeis A, Marmo F, Filomena Buonaguro E, Rossi R, Tomasetti C, Iasevoli F (2013). Imaging brain gene expression profiles by antipsychotics: region-specific action of amisulpride on postsynaptic density transcripts compared to haloperidol. Eur Neuropsychopharmacol 23: 1516-1529.

Desjardins S, Mayo W, Vallee M, Hancock D, Le Moal M, Simon H et al (1997). Effect of aging on the basal expression of c-Fos, c-Jun, and Egr-1 proteins in the hippocampus. Neurobiol Aging 18: $37-44$.

Deutch AY, Lee MC, Iadarola MJ (1992). Regionally specific effects of atypical antipsychotic drugs on striatal Fos expression: the nucleus accumbens shell as a locus of antipsychotic action. Mol Cell Neurosci 3: 332-341.

Dong SM, Kim YG, Heo J, Ji MK, Cho JW, Kwak BS (2009). YKP1447, a novel potential atypical antipsychotic agent. Korean J Physiol Pharmacol 13: 71-78.

Graff J, Rei D, Guan JS, Wang WY, Seo J, Hennig KM et al (2012). An epigenetic blockade of cognitive functions in the neurodegenerating brain. Nature 483: 222-226.
Guidotti A, Dong E, Kundakovic M, Satta R, Grayson DR, Costa E (2009). Characterization of the action of antipsychotic subtypes on valproate-induced chromatin remodeling. Trends Pharmacol Sci 30: 55-60.

Houston I, Peter CJ, Mitchell A, Straubhaar J, Rogaev E, Akbarian S (2013). Epigenetics in the human brain. Neuropsychopharmacology 38: 183-197.

Juergens RA, Wrangle J, Vendetti FP, Murphy SC, Zhao M, Coleman B et al (2011). Combination epigenetic therapy has efficacy in patients with refractory advanced non-small cell lung cancer. Cancer Discov 1: 598-607.

Kapur S (2003). Psychosis as a state of aberrant salience: a framework linking biology, phenomenology, and pharmacology in schizophrenia. Am J Psychiatry 160: 13-23.

Kazantsev AG, Thompson LM (2008). Therapeutic application of histone deacetylase inhibitors for central nervous system disorders. Nat Rev Drug Discov 7: 854-868.

Khan N, Jeffers M, Kumar S, Hackett C, Boldog F, Khramtsov N et al (2008). Determination of the class and isoform selectivity of smallmolecule histone deacetylase inhibitors. Biochem J 409: 581-589.

Kontkanen O, Lakso M, Wong G, Castren E (2002). Chronic antipsychotic drug treatment induces long-lasting expression of fos and jun family genes and activator protein 1 complex in the rat prefrontal cortex. Neuropsychopharmacology 27: 152-162.

Kwok JB (2010). Role of epigenetics in Alzheimer's and Parkinson's disease. Epigenomics 2: 671-682.

Lieberman JA, Bymaster FP, Meltzer HY, Deutch AY, Duncan GE, Marx CE et al (2008). Antipsychotic drugs: comparison in animal models of efficacy, neurotransmitter regulation, and neuroprotection. Pharmacol Rev 60: 358-403.

Lonergan E, Luxenberg J, Colford J (2002). Haloperidol for agitation in dementia. Cochrane Database Syst Rev CD002852.

Monti B, Polazzi E, Contestabile A (2009). Biochemical, molecular and epigenetic mechanisms of valproic acid neuroprotection. Curr Mol Pharmacol 2: 95-109.

Murphy CA, Feldon J (2001). Interactions between environmental stimulation and antipsychotic drug effects on forebrain c-fos activation. Neuroscience 104: 717-730.

Nagahara AH, Handa RJ (1997). Age-related changes in c-fos mRNA induction after open-field exposure in the rat brain. Neurobiol Aging 18: 45-55.

Numata S, Ye T, Hyde TM, Guitart-Navarro X, Tao R, Wininger M et al (2012). DNA methylation signatures in development and aging of the human prefrontal cortex. Am J Hum Genet 90: $260-272$.

Nuutinen T, Suuronen T, Kauppinen A, Salminen A (2010). Valproic acid stimulates clusterin expression in human astrocytes: implications for Alzheimer's disease. Neurosci Lett 475: 64-68.

Paxinos G, Franklin KBJ (2008). The Mouse Brain in Stereotaxic Coordinates, Compact. Elsevier: New York.

Peleg S, Sananbenesi F, Zovoilis A, Burkhardt S, Bahari-Javan S, Agis-Balboa RC et al (2010). Altered histone acetylation is associated with age-dependent memory impairment in mice. Science 328: 753-756.

Penner MR, Roth TL, Chawla MK, Hoang LT, Roth ED, Lubin FD et al (2011). Age-related changes in Arc transcription and DNA methylation within the hippocampus. Neurobiol Aging 32: 2198-2210.

Ricobaraza A, Cuadrado-Tejedor M, Perez-Mediavilla A, Frechilla D, Del Rio J, Garcia-Osta A (2009). Phenylbutyrate ameliorates cognitive deficit and reduces tau pathology in an Alzheimer's disease mouse model. Neuropsychopharmacology 34: 1721-1732.

Robertson GS, Matsumura H, Fibiger HC (1994). Induction patterns of Fos-like immunoreactivity in the forebrain as predictors of atypical antipsychotic activity. J Pharmacol Exp Ther 271: 1058-1066.

Semba J, Sakai M, Miyoshi R, Mataga N, Fukamauchi F, Kito S (1996). Differential expression of c-fos mRNA in rat prefrontal cortex, striatum, N. accumbens and lateral septum after typical 
HDAC inhibitors restore HAL response in aged mice JL Montalvo-Ortiz et al

and atypical antipsychotics: an in situ hybridization study. Neurochem Int 29: 435-442.

Semba J, Sakai MW, Suhara T, Akanuma N (1999). Differential effects of acute and chronic treatment with typical and atypical neuroleptics on c-fos mRNA expression in rat forebrain regions using non-radioactive in situ hybridization. Neurochem Int 34: 269-277.

Simonini MV, Camargo LM, Dong E, Maloku E, Veldic M, Costa E et al (2006). The benzamide MS-275 is a potent, long-lasting brain region-selective inhibitor of histone deacetylases. Proc Natl Acad Sci USA 103: 1587-1592.

Sumner BE, Cruise LA, Slattery DA, Hill DR, Shahid M, Henry B (2004). Testing the validity of c-fos expression profiling to aid the therapeutic classification of psychoactive drugs. Psychopharmacology (Berl) 171: 306-321.

Suzuki T, Uchida H, Takeuchi H, Nakajima S, Nomura K, Tanabe A et al (2009). Augmentation of atypical antipsychotics with valproic acid. An open-label study for most difficult patients with schizophrenia. Hum Psychopharmacol 24: 628-638.

Tang B, Dean B, Thomas EA (2011). Disease- and age-related changes in histone acetylation at gene promoters in psychiatric disorders. Transl Psychiatry 1: e64.
Tanic N, Perovic M, Mladenovic A, Ruzdijic S, Kanazir S (2007). Effects of aging, dietary restriction and glucocorticoid treatment on housekeeping gene expression in rat cortex and hippocampusevaluation by real time RT-PCR. J Mol Neurosci 32: 38-46.

Wadenberg ML (2010). Conditioned avoidance response in the development of new antipsychotics. Curr Pharmaceut Des 16: 358-370.

Wadenberg ML, Wiker C, Svensson TH (2007). Enhanced efficacy of both typical and atypical antipsychotic drugs by adjunctive alpha2 adrenoceptor blockade: experimental evidence. Int $J$ Neuropsychopharmacol 10: 191-202.

Watt ML, Rorick-Kehn L, Shaw DB, Knitowski KM, Quets AT, Chesterfield AK et al (2013). The muscarinic acetylcholine receptor agonist BuTAC mediates antipsychotic-like effects via the M4 subtype. Neuropsychopharmacology 38: 2717-2726.

Witta SE, Jotte RM, Konduri K, Neubauer MA, Spira AI, Ruxer RL et al (2012). Randomized phase II trial of erlotinib with and without entinostat in patients with advanced non-small-cell lung cancer who progressed on prior chemotherapy. J Clin Oncol 30: 2248-2255.

Zhao C, Li M (2010). c-Fos identification of neuroanatomical sites associated with haloperidol and clozapine disruption of maternal behavior in the rat. Neuroscience 166: 1043-1055. 